\title{
Segmentation of Multispectral Images of Works of Art Through Principal Component Analysis ${ }^{1}$
}

\author{
Stefano Baronti, Andrea Casini, Franco Lotti and Simone Porcinai \\ "Nello Carrara" Research Institute on Electromagnetic Waves IROE - CNR, \\ 64, Via Panciatichi, 50127 Florence, Italy
}

\begin{abstract}
Investigation of materials constituting painted layers of works of art (panels, canvas, frescoes) can be profitably done by means of non-destructive optical techniques based on the analysis of reflectance spectra in the visible and near infrared regions.

Accurate and high spectral resolution measurements can be obtained by means of fiber optics spectrophotometers, but only in small spot areas. Image spectroscopy systems can give instead a complete spectral information on the whole examined surface in a great number of bands, allowing direct visual interpretation. The analysis of such amount of data is not trivial. A possible approach is to decorrelate the data and concentrate the significant information in few images, by using principal component analysis (PCA).

In this work segmentation is investigated in order to partition the imaged scene into regions of spectral similarity to facilitate successive analysis. The results on a test tempera panel and on a predella painted in the XVI century show the effectiveness of the proposed approach, also revealing details undetectable by conventional techniques.
\end{abstract}

\section{Introduction}

Optical reflectance spectroscopy is an important non invasive technique in the field of preservation and restoration of paintings for the identification of the constituting materials (typically: preparation layers, pigments, varnishes, degradation products, etc.). Objective data can be gathered to monitor the conservation status of paintings.

After ten years of experience in this field, we may state that fiber-optic reflectance spectroscopy (FORS) in the visible and short wavelength infrared (400$2000 \mathrm{~nm}$ ) is almost always effective when a suitable archive of spectra is available as a reference $[1,2]$. To this purpose, an exhaustive archive of visible and nearinfrared reference spectra of the pigments used in the middle ages and renaissance florentine painting has been set up [4], on the basis of measurements on both pure pigment powders and test panels painted in accordance with ancient techniques.

Among non-invasive spectroscopic techniques, FORS is also the most functional, as it can be operated with transportable equipment and works with fiberoptic probes which can be easily handled to collect data from small spots on the painted surface. However, such a collection of local 1-D spectrograms (spectral resolution is $\sim \mathrm{nm}$ ), though rich in information to a chemist, cannot be readily correlated with the scene. Such a limitation makes 1-D reflectance spectroscopy less directly useful to restorers and art historians.

A technique which promises to be more efficient than FORS is its two-

${ }^{1}$ Work partially supported by the CNR Project Beni Culturali and by the EC project ERA: Environmental Research for Art Conservation, EV5V CT94 0548. 
dimensional extension: imaging spectroscopy (IS) or spectral imaging, which supplies very readable data by retaining the aspect of the objects. Imaging spectroscopes in the VIS-NIR regions are not yet available as handy transportable instruments of reasonable cost. For this reason we developed a transportable image spectroscopy system based on a Vidicon camera $(400-2000 \mathrm{~nm})$ and a set of several narrow-band interferential filters supervised by a computerized program that manages the whole acqusition process $[3,7]$.

The large amount of collected data (tenths of multispectral images of $754 \times 572$ pixels in the VIS-NIR range) has suggested to perform multivariate statistical analysis, such as principal component analysis (PCA), both to compress the meaningful information into few components and to segment the scene, mapping the areas with similar pigment composition. PCA has been already used in a variety of chemical applications and mapping of remotely sensed multispectral data [5-6].

In the first part of this work we report the results of the application of PCA to reflectance IS data acquired from a test tempera panel, after validation with data acquired using a high resolution spectrophotometer.

In the second part of the work an application is described to an ancient oil painted panel of XVI century: Luca Signorelli's Holy Trinity predella. Results showed that multivariate data analysis can help in the recognition of the materials, e.g. by allowing scene segmentation into areas with similar spectral behavior.

\section{Multispectral Image Acquisition}

The imaging setup used for the collection of the multiwavelength images has been assembled around a PbO-PbS Vidicon camera (Hamamatsu C2400-03). Spectral selection has been realized by means of narrow-band optical filters, lodged on 8-filter wheels, which are sequentially mounted in front of the camera lens [7].

A set of 29 narrow-band interferential filters has been used with full width at half maximum of $\sim 10 \mathrm{~nm}$ and $0.01 \%$ blocking factor out of band (from X-ray to far infrared). Illumination is supplied by two projectors symmetrically placed at the camera sides with $\sim 45^{\circ}$ beam incidence angles. The illuminance in the visible is $\sim 12000$ lux. In the UV the irradiance is very low $\left(<0.4 \mathrm{~W} / \mathrm{m}^{2}\right)$, while in the IR (up to $\sim 2.5 \mu \mathrm{m}$ ) it is $67 \mathrm{~W} / \mathrm{m}^{2}$. In order to normalize the amplitude of the collected images, five SPECTRALON ${ }^{\circledast}$ reflectance standards are included in the scene: two of the standards (99\% and 3\% reflectance) are used for the normalization according to a linear model of the camera response (after gamma correction). The other three standards $(75 \%, 50 \%, 10 \%)$ are used to control the quality of the measurement.

\section{Principal Component Analysis}

PCA is a method which gives a compressed description of the data variance by means of a small number of uncorrelated variables. In our case, each of the $n$ collected spectra (objects) may be represented by a point in a $v$-dimensional space, where $v$ is the number of wavelengths (variables). PCA involves orthogonal rotation of the original axes, each representing an original variable, into new axes. The new axes, called Principal Components, are the eigenvectors of the data variancecovariance matrix $[8,9]$. PCA allows the reduction of the parameter space 
dimensionality, as the number of new axes $a$ (variables) needed to describe most of the sample data variance is usually much less than $v$.

\section{Analysis of the Test Panel}

The test panel is a wooden tablet prepared with a gypsum layer and painted with four pigments using a tempera medium. Formulas and dominant wavelengths of the four pigments are reported on the Table I. Three rectangular stripes, for each pigment, were painted using the pure pigment and two mixtures with $10 \% \mathrm{w} / \mathrm{w}$ and $5 \%$ w/w carbon black, thus creating 12 stripes of the same size differentiated either for their dominant wavelength $\left(\lambda_{D}\right)$, due to the nature of the pigment, or for their brightness, due to the carbon black percentage. Moreover, some carbon black lines had been drawn on the gypsum layer, in order to simulate the presence of underdrawings. The purity of the pigments was checked by means of X-ray diffraction; the average dimension of the carbon black particles, measured by laser scattering granulometry is about $10 \mu \mathrm{m}$.

\begin{tabular}{|c|c|c|}
\hline PIGMENT & FORMULA & $\lambda_{\text {n }}(\mathrm{nm})$ \\
\hline cinnabar & $\mathrm{HgS}$ & 619 \\
\hline malachite & $\mathrm{Cu}_{2} \mathrm{CO}(\mathrm{OH})_{2}$ & 510 \\
\hline yellow ochre & $\mathrm{FeO}(\mathrm{OH})$ & 583 \\
\hline chromium oxide & $\mathrm{Cr}_{2} \mathrm{O}_{3}$ & 560 \\
\hline
\end{tabular}

Table I: Formula and dominant wavelength $\left(\lambda_{D}\right)$ of the four pigments of the test tablet.

Table II reports the contributions of the first five PCs to the overall variance as a result of the application of PCA to 29 images of the tablet almost equally distributed in the VIS-NIR region (420-1550 nm). It appears that the first five PCs account nearly entirely for the total variance $(99.62 \%$ ) of the data set.

\begin{tabular}{|c|c|c|}
\hline PCs & Var. \% & Cumul. Var \% \\
\hline 1 & 86.83 & 86.83 \\
\hline 2 & 6.66 & 93.49 \\
\hline 3 & 4.23 & 97.72 \\
\hline 4 & 1.2 & 98.92 \\
\hline 5 & 0.7 & 99.62 \\
\hline
\end{tabular}

Table II: Variance and cumulative variance for the first five principal components from 29 VIS-NIR images of the test tablet.

The redistribution of variance has an interesting theoretical interpretation: if the variance is considered as a measure of the information content, then a packing of information has occurred because a lower number of variables contains a large percentage of the total data variance. In our case, most of the information is expected to be contained in the five largest components.

Figure 1 reports the first four $\mathrm{PC}$ images of the tablet. The gray scale value of the pixels was obtained by scaling from 0 to 255 their coordinates in the $\mathrm{PC}$ domain.

It can be noted that in the $\mathrm{PC} 1$ image the gray values of the areas corresponding 
to pure pigments are generally higher than those corresponding to mixtures with carbon black. However, the clustering in the $\mathrm{PC}$ domain of points belonging to the twelve differently painted zones on the tablet can be better appreciated by considering the 2-D score plots. These are the projections of the position vectors (in the PC space) of the objects (pixel spectra) into a 2-dimensional subspace. The score plot which offers the clearest clustering of the 12 painted zones is the projection on the PC1/PC3 plane, as shown in Figure 2, where the pixel densities are represented by their level lines. It is possible to appreciate not only the different pigments but also the zones with the same pigment and different percentages of carbon black.

The study of the trend of the eigenvectors versus wavelength can be helpful in understanding the common features of the groups and why an object (a spectrum of a pixel in our case) is located in a specific position on the score plot. The weight plot reported in Figure 3 shows how the different wavelengths influence the PC1 and PC3 eigenvectors. PC1 eigenvector receives a contribution from the nearinfrared bands $(83.8 \%)$ heavier than from the visible ones $(16.2 \%)$; the disposition of the weights on the same plot shows that all the near-infrared bands have a positive and almost equal weight on the $\mathrm{PCl}$. Approximately $\mathrm{PCl}$ lies in the subspace defined by the NIR bands; furthermore, as these bands give $\mathrm{PC} 1$ almost the same contribution, $\mathrm{PC} 1$ is oriented mainly along the diagonal of this subspace.

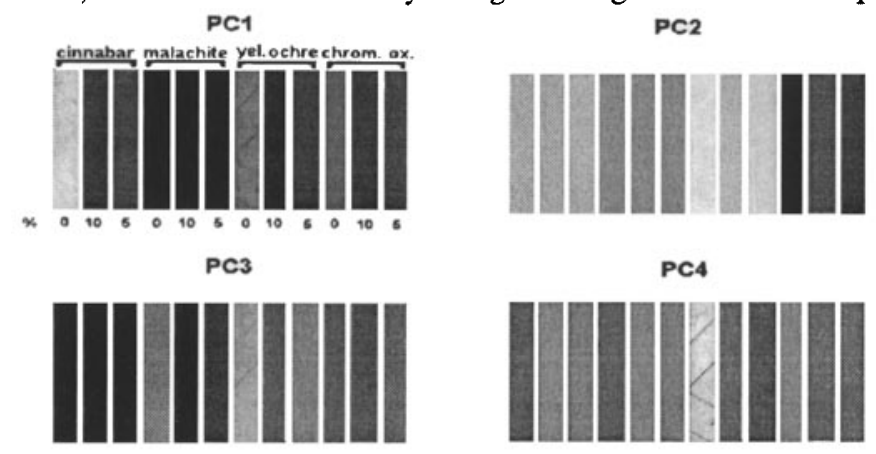

Figure $1-P C 1, P C 2, P C 3$ and PC4 images of the test tablet from a sequence of 29 VIS-NIR images. Carbon black percentages are indicated.

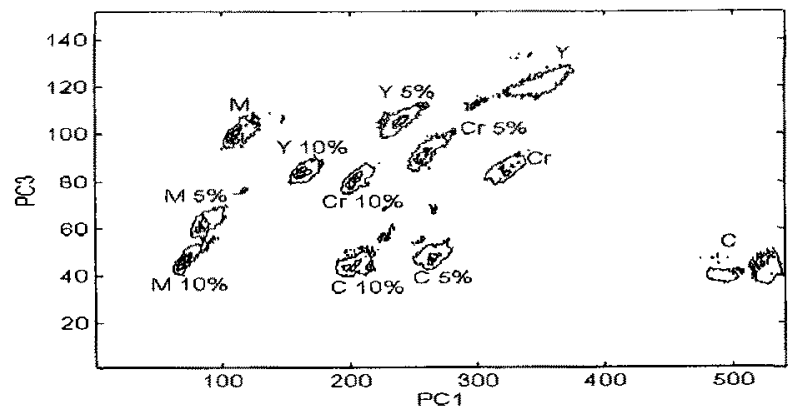

Figure 2 - Score plot PC3/PC1 from PCA on 29 VIS-NIR images of the test tablet.C: cinnabar; Y: yellow ochre; $M$ : malachite; Cr: chromium oxide. The fractions carbon blackpigment $(w / w)$ are reported as percentages. 


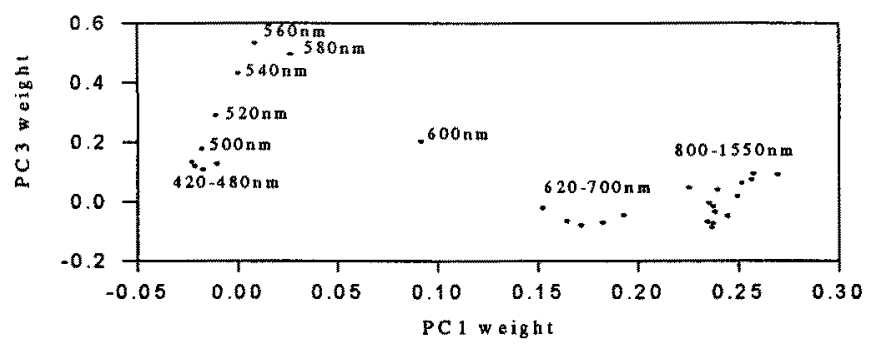

Figure 3 - Weight plot PC3/PC1 from PCA on 29 VIS-NIR images of the test tablet.

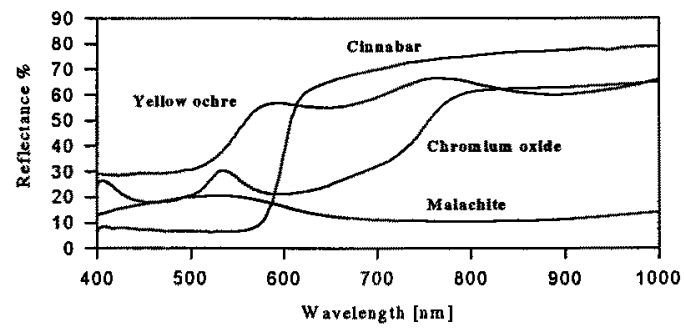

Figure 4 - Spectra of the four pure pigments of the test tablet taken with FORS.

In other words, the most important contribution to variance is given by the spread of the NIR mean reflectances. Therefore, as carbon black reflectance has a constant high absorption through the NIR region, its percentage determines the mean NIR reflectances of the mixtures and the separation of each pigment group into clusters along the PC1 axis (Figure 2). The visible bands in the range 420-600 $\mathrm{nm}$ have a strong influence $(92.7 \%$ ) on the third principal component. In particular, the bands at $560 \mathrm{~nm}$ and $580 \mathrm{~nm}$ give the highest contribution for this test tablet. Among the four pigments, yellow ochre and cinnabar receive the greatest discrimination just by the wavelengths in the range $560-580 \mathrm{~nm}$, (Figure 4); indeed, these pigments are placed at the extrema of the PC3 range in the PC1/PC3 score plot (Figure 2).

It is interesting to note how underdrawings, which are usually made of carbon black, are clearly revealed on PC1 and PC4 images (Figure 1), especially under yellow ochre, which is characterized by a low absorption in the NIR region. Underdrawings become visible in the NIR images because the radiation in this wavelength range penetrates more deeply into the painted layers. It is partially absorbed by the carbon black, which has almost constant low reflectance, and partly reflected by the gypsum background layer, which has high reflectance (but for few well defined characteristic absorption bands). 


\section{Analysis of a XVI Century Painted Panel}

The results obtained on the test tablet furnished some guidelines for the application to works of art. The above explained methodology has been profitably applied to the study of a scene of the Holy Trinity predella (on exhibit at the Uffizi Gallery), which was painted by Luca Signorelli in the early years of XVI century. It is an oil painted panel of $32 \times 204 \mathrm{~cm}$, divided into three scenes of Jesus's passion. A sequence of 29 multispectral images of Flagellation scene was acquired by our image spectroscopy system in the visible and near-infrared region. In Figure 5 we report the plot of the first eigenvector (PC1) after application of PCA to this set of images. It can be seen that the main contribution to the total variance of the data set is given by the NIR bands, which have similar weight, showing that the variance accounted from them is mainly due to the variations of the average reflectances

The high penetration of NIR radiation suggests to evaluate the spectral behaviour of the pixels in this region, to evidence information from deeper layers. To this purpose, and to avoid that higher variations in the visible range could mask those in the NIR, we limited the PCA to a subset of 13 NIR images $(800-1550 \mathrm{~nm})$.

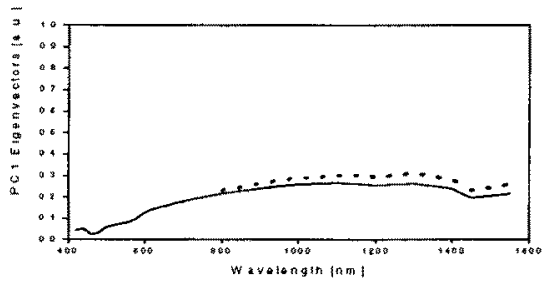

Figure 5 - PCl Eigenvectors from PCA on 29 VIS-NIR images(solid) and on 13 NIR images (dotted) of the predella.

In Figure 6 the first three PC images are reported which account for more than $99 \%$ of the variance in the NIR data set. Again, the variation of the mean reflected light gives the most important contribution to data variance $(91.3 \%$ accounted by PC1). In fact, the most important eigenvector (PC1) is now uniformly weighted across all the spectral bands (Figure 5). In general we are not interested in this variance which gives poor information concerning chemical properties of the materials and does not help in segmentation. To eliminate this variance, we normalized the reflectance values of each multispectral image $\mathrm{I}(\mathrm{x}, \mathrm{y}, \lambda)$ to the image at $800 \mathrm{~nm} \mathrm{I}(\mathrm{x}, \mathrm{y}, \lambda=800)$ before performing PCA:

$$
I_{\text {norm }}(x, y, \lambda)=I(x, y, \lambda)-I(x, y, \lambda=800)
$$

This normalization has the effect of bringing to zero the first element of the eigenvectors associated with each component. Adopting this normalization, PC1 generally accounts for the variance of the baseline slope of the reflectance spectra.

The variance, computed by applying PCA to the normalized data (NIR range) resulted $87 \%$ for $\mathrm{PC} 1,9.42 \%$ for $\mathrm{PC} 2$ and 0.91 for $\mathrm{PC} 3$.

The normalized eigenvector $\mathrm{PC} 1_{\text {norm }}$ is highly correlated with the nonnormalized eigenvector PC2 (Fig.7) and the same happens for the PC2 norm and PC3. 

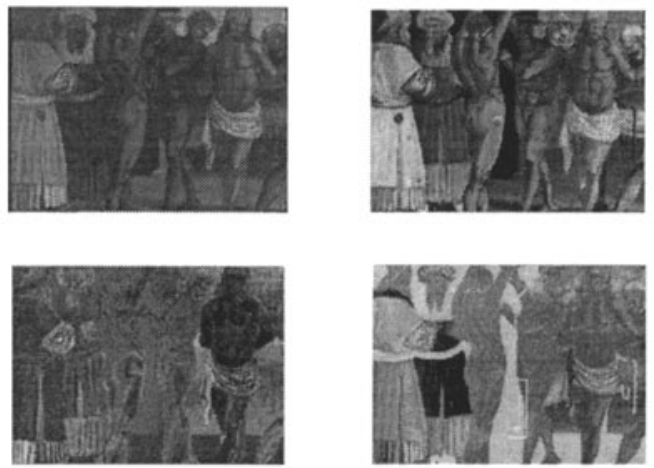

Figure 6 - Detail of Luca Signorelli's Holy Trinity predella. Clockwise, from top left: grey level representation, $P C 1$ image, $P C 2$ image, $P C 3$ image.

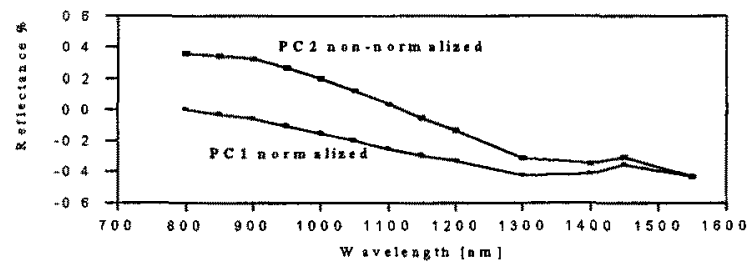

Figure $7-P C 1$ and $P C 2$ eigenvectors from PCA on 13 NIR images of the predella.

The correlation coefficient $\mathrm{r}$ between the normalized $\mathrm{PCi}_{\mathrm{norm}}$ and non-normalized PC(i+1) eigenvectors decreases with $i$ because the variance subtracted adopting this normalization is not only due to the energy offset in the reflectance spectra of pixels.

$$
\mathrm{r}\left(\mathrm{PC}_{\text {norm }}, \mathrm{PC} 2\right)=0.994 ; \quad \mathrm{r}\left(\mathrm{PC} 2_{\text {norm }}, \mathrm{PC} 3\right)=0.67
$$

The PCs, now accounting for the variance due to finer spectral features of the pixel spectra, become more important in the $\mathrm{PC}$ model and allow to evidence zones characterized by different chemical composition of the materials.

Visual inspection of the first two PC images (Figures 8) reveals that the images obtained from the normalized variance-covariance matrix have better contrast and appear to be more useful for visual segmentation purposes. In particular, by means of the PC1 and PC2, it is possible to distinguish zones which appear to be painted with the same pigment. For example in the PC1 image (Figure $8 \mathrm{~A}$ ), a pilaster behind a whipper appears to be split into two distinct parts. The left part of the pilaster had been likely painted as correction of the original profile, and covers a portion of the dark wall. In fact, the subsequent observation of the reflectance spectra in the NIR region shows lower values of reflectance in the left part than in the right one. Moreover, in the PC2 image (Figure $8 \mathrm{~B}$ ), the values of Jesus Christ's skin are quite different from the other bodies present in the scene, indicating that the artist used different pigment compositions, not evident on a visual examination. 


\section{Conclusions}

Image Spectroscopy in the visible and near-infrared regions, in conjunction with PCA can be profitably used for segmenting painted zones characterized by similar spectral behavior and therefore to map areas according to their chemical composition or physical properties. The application of multivariate image analysis to the image sequence of the test tablet showed that it is possible to obtain a complete segmentation of zones homogeneously painted.

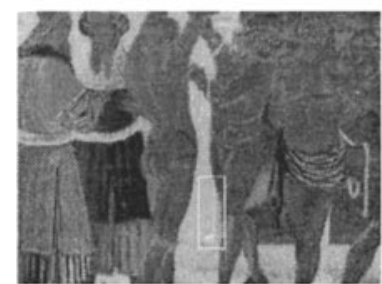

A

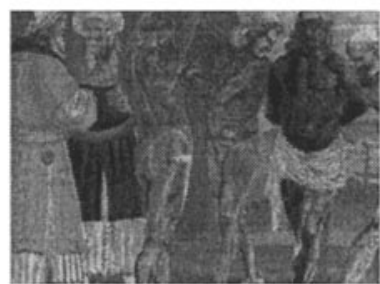

$\mathrm{B}$

Figure 8 - Normalized image of the predella from PCA on 13 NIR images. A PC1; B: PC2.

Non-invasivity and objectivity of the instrumentation are the main features that make this procedure particularly suitable for investigating paintings both to monitor their status of conservation and to guide restoration activity. From the results obtained on the Holy Trinity predella by Luca Signorelli, it appears that the use of image spectroscopy together with Principal Component Analysis is a powerful tool in investigating the pigment distribution on paintings.

Segmentation maps can be easily done, grouping areas with similar spectral behaviour, which help both in the interpretation of the painting and in the selection of minimal but meaningful micro-sampling, when destructive analysis is required.

\section{Acknowledgments}

Thanks are given to Mr. L. Stefani (IROE) for his technical support during the measurements and to Dr. M. Picollo for helping in the FORS measurements.

\section{References}

1 M. Bacci, Sensors and Actuators B 29, 190 (1995).

2 A. Orlando, M. Picollo, B. Radicati, S. Baronti and A. Casini, Appl. Spectrosc. 49, 459, (1995)

3 M. Bacci, S. Baronti, A. Casini, F. Lotti, M. Picollo and O. Casazza, Material Issues in Art and Archaeology III 267, 265-282 (1992).

4 R. Linari, M. Picollo and B. Radicati: IROE Technical report TR/POE/92.7, July, 1992

5 P.J. Ready and P.A. Wintz, IEEE Trans. Commun. 21, 1123-1130 (1973)

6 M.E. Kargacin and B.R. Kowalski, Anal. Chem. 5, 2300, (1986)

7 A. Casini, F. Lotti, M. Picollo, L. Stefani and G. Troup: Proc, Atti Fondaz. G. Ronchi, LI, n.1-2, pp.289-303 (1996).

8 K.V. Mardia and J.T. Kent and J.M. Bibby: Multivariate Analysis. Birnbaum and Lukacs London, 1979.

9 H. Martens and T. Nes: Multivariate Calibration (Wiley and Sons, New York, 1989). 